\title{
Konstituierung der sozialkritischen pädagogischen Richtung zwischen den beiden Weltkriegen in Slowenien ${ }^{1}$
}

\author{
Edvard PROTNER
}

\begin{tabular}{l} 
ARTICLE INFO \\
\hline Article history: \\
Received 19 March 2019 \\
Accepted 6 March 2020 \\
Available online 31 July \\
2020 \\
\hline Keywords: \\
Slovenia, 1918-1941, \\
marxism, pedagogical \\
pluralism, empirical \\
research \\
\hline E. Protner \\
University of Maribor • \\
Faculty of Arts \\
Department of \\
Pedagogy • \\
Koroška cesta 160 \\
2000 Maribor • \\
Slovenija $\bullet$ \\
edvard.protner@um.si
\end{tabular}

\begin{abstract}
The Constitution of a Socio-critical Pedagogical Direction in Slovenia between the World Wars

The period between the World Wars in Slovenia is characterized by a pronounced pluralism of pedagogical directions and concepts. While the art (Geisteswissenschaftliche) pedagogical direction was consolidated at the university, the reform pedagogical direction (Reformpädagogik) was increasingly used in professional associations of teachers. Within this movement and in parallel, the questions of social justice and the ideological orientation of education have become increasingly at the forefront. The initiative in this field was taken over by marxist teachers, but the socio-critical pedagogical concept was not unambiguously tied to the communist ideological background. Within the framework of the socio-critical pedagogical direction, empirical pedagogical research began to develop in Slovenia.
\end{abstract}

Die Zeit zwischen den beiden Weltkriegen zeichnete sich in Slowenien durch einen ausgeprägten Pluralismus pädagogischer Richtungen und Konzepte aus. Auch wenn der Herbartianismus zu dieser Zeit theoretisch schon überwunden war, spiegelte die Schulrealität immer noch den Geist der ,alten Schule“. Während sich zur Zeit der Gründung der ersten slowenischen Universität in Ljubljana die geisteswissenschaftliche Pädagogik etablierte (Karel Ozvald und Stanko Gogala waren zwei repräsentative slowenische Vertreter dieser Richtung), setzte sich in den Lehrerverbänden die reformpädagogische Richtung durch. Innerhalb dieser Bewegung, aber auch parallel dazu, traten immer häufiger Fragen nach der sozialen Gerechtigkeit und der ideologischen Ausrichtung des Schulwesens in den Vordergrund. Die Konstituierung der sozialkritischen pädagogischen Richtung ${ }^{2}$ hing eng mit

\footnotetext{
${ }^{1}$ Übersetzung: Brigita Kacijan.

Der Artikel entstand (auch) im Rahmen der Programmgruppe »Slowenische Identität und Kulturbewusstsein in sprachlichen und ethnischen Kontakt Räumen in der Vergangenheit und in der Gegenwart«, Nr. P6-0372, die durch die Slowenische forschung Agentur finanziert werde.

${ }^{2}$ Slowenien war bis 1918 ein Teil der österreichisch-ungarischen Monarchie, weswegen sich auch nach der Entstehung des Königreichs der Serben, Kroaten und Slowenen die traditionelle pädagogische Sichtweise hielt, die in erster Linie im zentraleuropäischen deutschen Sprachraum entstanden war. Es wird davon ausgegangen,
} 
der Entwicklung der Arbeiterbewegung und der marxistischen Gesellschaftskritik zusammen (vgl. Protner, 2000). Ihre Vertreter betrachteten die Bildung als sozialen Prozess des Hineinwachsens des Individuums in die Gesellschaft - die Bildungsziele wurden aus der Analyse der sozialen Bedingungen, in denen das Kind aufwächst, und aus der (größtenteils) marxistischen Vision der gesellschaftlichen Entwicklung abgeleitet. In Slowenien waren die prominentesten Vertreter dieser pädagogischen Richtung linksorientierte Lehrer. Die jüngere Generation organisierte in den 30er Jahren eine Bewegung mit dem Namen Lehrerbewegung (učiteljski pokret) - ihre Vertreter hoben, noch stärker als die älteren Kollegen, die Notwendigkeit empirischer Studien und der Darstellung der miserablen sozialen Lebensverhältnisse der Arbeiter und Bauern als Mittel zur Kritik der damaligen Gesellschaftsordnung hervor. Sie gingen von der Prämisse aus, dass eine Schulreform nicht erfolgreich sein kann, wenn zuvor nicht die soziale, wirtschaftliche und kulturelle Situation und das damit verbundene politische System verbessert wird.

Im Beitrag werden die Hauptvertreter dieses pädagogischen Konzepts in Slowenien und ihre Aktivitäten vorgestellt, außerdem werden die Anfänge der empirischen pädagogischen Forschung im Rahmen dieses Konzepts hervorgehoben.

\section{Franjo Žgeč und die Anfänge des sozialkritischen Paradigmas in Slowenien}

Der Initiator der sozialkritischen Pädagogik in Slowenien war Franjo Žgeč. Er erlebte die sozialistische Oktoberrevolution in Russland unmittelbar als Kriegsgefangener, wo er mit Ideen in Kontakt kam, die seine sozialkritische Ausrichtung beeinflussten. Er lernte zu dieser Zeit nicht nur die Ideen des Sozialismus kennen, sondern auch das pädagogische Konzept der

dass ein fachlich versierter Leser problemlos den Herbartianismus, die geisteswissenschaftlichen Pädagogik und die Reformpädagogik als allgemein bekannte und theoretisch gut beschriebene Richtungen der Pädagogik wiedererkennen kann, wobei er sich vermutlich fragen wird, aufgrund welcher Kriterien sie hier zugeordnet wurden. Größere Schwierigkeiten könnte der Begriff sozialkritische Pädagogik verursachen, da diese Bezeichnung außerhalb Sloweniens nicht geläufig ist. Es geht um ein Konzept, das der im deutschsprachigen Raum der „Materialistischen Erziehungstheorie“ (Schmied-Kowarzik, 1983), „Materialistisch orientierten Pädagogik“ (Tenorth, 1992), „Historisch-materialistischen Pädagogik“ (Krüger, 1997), meist aber der „Marxistischen Pädagogik“ ähnlich ist. Bei diesen Bezeichnungen ist das Klassifikationskriterium der ideologische bzw. philosophische Hintergrund des pädagogischen Konzepts und genau dieses Kriterium unterscheidet sich von den Kriterien, nach denen die herbartianische, reformistische und geisteswissenschaftliche pädagogische Richtung klassifiziert werden. Die Diskussion stützt sich auf die Klassifizierung, die von Zdenko Medveš (2015) in Slowenien eingeführt und etabliert wurde. Als gemeinsamen Nenner bei der Kennzeichnung pädagogischer wissenschaftlicher Paradigmen berücksichtigt Medveš die Struktur des Bildungsprozesses, den Zusammenhang zwischen den Bildungsfaktoren und welchem der Bildungsfaktoren (Bildungsmedium) die entscheidende Rolle im pädagogischen Handeln zugeschrieben wird. Dabei geht es um drei Aspekte, in der Unterrichtstheorie als didaktisches Dreieck (Lehrkraft, Lernender, Lernstoff) bekannt ist, dem er noch den vierten, in der pädagogischen Theorie bekannten Aspekt des Lebensumfelds hinzufügt. Auf dieser Grundlage entwickelt er die folgende Klassifikation der pädagogischen Paradigmen (Richtungen): „Vier Medien ermöglichen es, vier pädagogische Paradigmen bzw. Theorien aufzustellen. Die reformatorische oder autopoetische Pädagogik der Systemtheorie (Luhmann) versteht das Kind als Bildungsmedium, der Herbartianismus sieht den Erzieher/Lehrer (in seiner Persönlichkeit) als sein Medium, die geisteswissenschaftliche oder Kulturpädagogik versteht den Inhalt mit einer entsprechenden geistig, wertebezogen und kulturell wertvollen Botschaft als ihr Medium während die sozialkritische Pädagogik das Lebensumfeld (das soziale und materielle Umfeld), den Kontext oder Raum als ihr Medium entwickelt" (Ebda, S. 14). 
produktiven Schule (eines der Konzepte der Reformpädagogik), das vom russischen Pädagogen Blonskij entwickelt wurde (Tancer, 1991). Beides zeigte sich in seinem pädagogischen Konzept, das er 1923 in seinem Buch Probleme der Erziehung der breitesten Schichten unseres Volkes (Žgeč, 1923) veröffentlichte. Dieses Konzept war auch das Thema seiner Doktorarbeit, wofür er einen Preis des Königs bekam, der es ihm ermöglichte, einen einjährigen Studienaufenthalt (1923/24) bei Prof. Simon (Laboratorium für experimentelle Pädagogik) an der Sorbonne in Paris wahrzunehmen. Nach seiner Rückkehr aus Paris hielt er sich einige Zeit am Rousseau-Institut in Genf auf, wo er einige der führenden Persönlichkeiten der Reformpädagogik (Claparéde, Dottrens, Ferriere) und ihre Ansichten kennenlernte (Tancer, 1991, S. 10), was den Kern seines pädagogischen Denkens stark prägte.

Das konzeptionelle und praktische Programm des Lehrerverbands Pädagogische Zentrale in Maribor war für die Etablierung seiner Sichtweise unter den slowenischen Lehrern von großer Bedeutung (Žgeč, 1928). Auch hier kommt seine Sympathie für die Reformpädagogik und die sozialkritische Pädagogik zum Ausdruck. Der zweite Programmpunkt besagt, dass die Pädagogische Zentrale Erziehungsprobleme sowohl auf psychologisch-pädagogischer als auch auf soziologischer Ebene lösen werde. Beide sollen dabei auf modernen wissenschaftlichen Grundlagen basieren.

Es lässt sich nur schwer rekonstruieren, welche pädagogischen Einflüsse aus dem Ausland die Entstehung des sozialkritischen pädagogischen Konzepts maßgeblich mitprägten. Zweifellos kannten die Mitglieder der Pädagogischen Zentrale Kanitz’ Buch „Das proletarische Kind in der bürgerlichen Gesellschaft“" $(1925)^{3}$. Erwähnt werden sollte allerdings auch, dass Ideen, die denen von Kanitz ähneln, auch bei einheimischen Vertretern der sozialkritischen Pädagogik zu finden waren.

Kanitz analysierte in diesem Werk auf der Grundlage soziologischer und psychologischer Analysen die untergeordnete Lage der proletarischen Kinder in Wirtschaft, Familie und Recht sowie im Verhältnis zu den Erwachsenen. Er schlussfolgerte: „Auch hier zeigt sich die Korrektheit der marxistischen Behauptung, dass die Produktionsart die Grundlage ist, auf der die anderen kulturellen Bereiche aufbauen. Durch die Erforschung der Situation der Kinder im wirtschaftlichen Leben und der daraus hervorgehenden Lage der Kinder in der Familie, im Rechtswesen, im alltäglichen Leben, kurz gefasst in allen kulturellen Bereichen, eröffnet sich, meiner Meinung nach, ein außerordentlich fruchtbares Forschungsfeld für die marxistische gesellschaftliche Vision [...]“ (Ebda, S. 91).

Gerade die Konsolidierung der marxistischen sozialen Ansichten stand im Vordergrund der Bemühungen der Vertreter der sozialkritischen Pädagogik und diese Aufgabe sollte von der

\footnotetext{
${ }^{3}$ In der Universitätsbibliothek in Maribor gibt es eine Kopie dieses Buches, das den Stempel des Pädagogischen und didaktischen Zirkels (Vorläufer der Pädagogischen Zentrale) Maribor trägt, was beweist, dass es sich um eine Kopie handelt, die den Mitgliedern der Pädagogischen Zentrale zwischen den beiden Weltkriegen in der Verbandsbibliothek zugänglich war (diese galt als die größte pädagogische Bibliothek im ehemaligen Jugoslawien). Deutschkenntnisse waren unter den slowenischen Lehrern damals durchaus üblich.
} 
soziologischen Ausbildung der Lehrer übernommen werden. Die erste derartige entschlossene Initiative entstand in Slowenien im Jahr 1926, als die Mitgliederversammlung der Vereinigung der jugoslawischen Lehrerschaft (UJU), Kommissariat Ljubljana, die Deklaration zur Entpolitisierung und Vereinheitlichung der fachpolitischen Ausrichtung der slowenischen Lehrerschaft verabschiedete. Im folgenden Jahr wurde im Rahmen der Vereinigung UJU der Zentralausschuss für Selbstbildung gegründet, der die Arbeit der Selbstbildungsarbeitskreise in den verschiedenen Lehrerverbänden koordinieren und jährlich einen Fortbildungskurs in Ljubljana organisieren sollte. Linksorientierte Lehrer, die die Deklaration am vehementesten unterstützten, sahen in diesen Selbstbildungskreisen eine gute Gelegenheit für eine ideologische, politische und fachliche Beeinflussung der Lehrerschaft.

Einer der Beschlüsse des ersten Selbstbildungskurses in Ljubljana war, „dass eine grundlegende soziologische Ausbildung der Lehrerschaft unerlässlich ist. Ohne Kenntnisse der Soziologie sucht sich der Lehrer mit verbundenen Augen seinen Weg durch die Gesellschaft“" (Pahor, 1927, S. 45).

Im Zusammenhang mit der inhaltlichen Ausrichtung der Selbstbildungsaktionen entwickelte sich ein Jahr später eine heftige Kontroverse, bei der sich drei Lager herauskristallisierten: Das erste Lager befürwortete die Soziologie bzw. die Sozialwissenschaften als das grundlegende Wissen, das Lehrer unbedingt benötigten und sie in ihrer Ausbildung an der Lehrerbildungsanstalt nicht bekommen hatten. Vertreter der Reformpädagogik forderten eine größere Betonung der Psychologie, während das dritte Lager für eine Vertiefung des pädagogischen Wissens plädierte.

An der Kontroverse nahm auch Žgeč teil, der trotz seiner weltanschaulichen und politischen Überzeugung eine ziemlich neutrale Haltung einnahm. Žgeč hatte sofort nach seiner Rückkehr aus der Kriegsgefangenschaft Kontakt zur Kommunistischen Partei in Ljubljana gesucht und in seinem Heimatdorf eine Parteizelle gegründet. Seine Parteiaktivitäten setzte er auch als Student illegal an der Universität in Ljubljana fort. Dennoch kann man seinen pädagogischen Bemühungen keine politische Motiviertheit zuschreiben, die auf direkten Parteianweisungen basieren würden (vgl. Protner, 2000). Es ging ihm in erster Linie darum, die Soziologie als pädagogische Hilfswissenschaft zu etablieren, die den Lehrer zu einer selbstständigen und kritischen Bewertung der Rolle der Erziehung in der Gesellschaft befähigt und es ihm ermöglicht, die sozialen, wirtschaftlichen, kulturellen, hygienischen und anderen Bedingungen, die eine erfolgreiche Erziehungsarbeit verhindern, zu erkennen. Dabei reichte Žgeč nicht ein theoretisches Studium, sondern er sah auch Feldforschung vor, die empirische Daten liefern würde, die für die spezifische ländliche Umgebung Sloweniens gültig waren. Zu diesem Zweck entwickelte er die Fragebögen des Entwicklungsstands der Schüler (sie wurden 1926 von der Pädagogischen Zentrale in Maribor herausgegeben), mit denen er versuchte, eine abhängige Verbindung zwischen dem sozialen und kulturellen Umfeld des heranwachsenden Kindes und seinem körperlichen und geistigen Entwicklungsstand 
herzustellen. Das war in Slowenien der erste Versuch empirische pädagogisch-soziologische Forschung $\mathrm{zu}$ betreiben, was auch eine radikale Neuheit im methodologisch-epistemologischen Paradigma der pädagogischen Forschung darstellte. Žgeč war sich von Anfang an der Einschränkung dieser Art von Forschung bewusst, denn er schrieb in der Interpretation der so gewonnenen Daten: „Ich gebe zu, dass ich den Unterschied in der körperlichen und geistigen Entwicklung unseres Kindes in verschiedenen Milieus beweisen und auch aus diesem Blickwinkel die Freiheit der Schule in ihrem Handeln rechtfertigen möchte und zwar die weitest und tiefest mögliche Autonomie der Schule, aber dazu bedarf es einer umfangreicheren Studie. Es wäre zu früh, aus unseren gesammelten Daten weitreichende Schlussfolgerungen zu ziehen“ (Žgeč, 1926, S. 56-57).

Bereits 1928 ging Zgeč erneut die empirische Forschung an. Diesmal versuchte er mit Hilfe der in der Pädagogischen Zentrale organisierten Lehrer sowie ausgefüllter Beobachtungsblätter die Erforschung des Einflusses der sozialen, wirtschaftlichen, kulturellen und hygienischen Umstände auf die Entwicklung der Kinder zu verbreiten und zu verbessern. In den neuen Beobachtungsblättern, die die Lehrer ausfüllen sollten, widmete er seine besondere Aufmerksamkeit dem Einfluss der Wohnverhältnisse, Ernährung, Kleidung und auch dem Einfluss von Alkoholismus, Armut und anderen Umständen auf die geistige Entwicklung, den Schulerfolg und eventuell auftretende Besonderheiten im Verhalten der slowenischen Kinder. Allerdings stieß er beim Datensammeln auf zahlreiche Hindernisse. Von den freiwillig mitarbeitenden Lehrern verlangte die Arbeit viel Zeit und auch Ressourcen. Außerdem war das Sammeln der Daten im statistischen Sinne mangelhaft geplant (Vgl. Bergant, 1962). Trotz aller Schwierigkeiten und Unzulänglichkeiten entstanden auf der Grundlage der gesammelten Daten einige Studien mit einem besonders sozialkritischen Schwerpunkt.

Zwei typische Beispiele sind die Beiträge von A. Šuligoj (1928 und 1929) mit den Titeln Einfluss der Wirtschafts- und Wohnbedingungen auf die Entwicklung der Kinder und Wohnbedingungen und ihr Einfluss auf das Kind sowie das Buch von K. Doberšek (1929) Einfluss der sozialen Verhältnisse auf die Kinder in Prevalje, das schon im Titel die Ausrichtung suggeriert. Auf empirischer Forschung gründet auch das Buch von J. Jurančič (1930) Aus der Schule für die Nation. Seine Arbeiten entstanden während der immer größer werdenden Wirtschaftskrise und den sich verschärfenden politischen Verhältnissen. Diesbezüglich ist es wichtig zu erwähnen, dass alle erwähnten Autoren Mitglieder oder zumindest Sympathisanten der Kommunistischen Partei waren und mit ihrer ideologischen Ausrichtung sehr stark die jüngeren, linksorientierten Lehrer beeinflussten, die sich in der zweiten Hälfte der 30er Jahre zur sog. Lehrerbewegung zusammenschlossen. 


\section{Zweite Phase der Entwicklung des sozialkritischen pädagogischen Paradigmas in Slowenien}

Die ersten Programmrichtlinien für die Arbeit der Lehrerbewegung entstanden 1936, als die Machthaber die Aktivitäten der Vereinigung junger Intellektueller in Maribor verboten, in der junge arbeitslose Lehrer am zahlreichsten vertreten und am aktivsten waren. Šegula (1974) erinnert sich daran, dass damals verschiedene Berufsverbände und Weltanschauungsverbände versuchten, die arbeitslosen Lehrer unter ihrem Einfluss zu halten. Um ihren Einfluss auf die arbeitslosen Lehrer zu erhalten und zu erhöhen, unterstützten auch linksorientierte Lehrer die Gründung einer Abteilung arbeitsloser Lehrerabsolventen im Rahmen des städtischen Lehrerverbands Maribor und dies kann als der Ursprung der Lehrerbewegung verstanden werden. Die Besonderheit ihrer Aktionen lag, laut Strmčnik (1980, S. 84), darin, dass sie „gesellschaftspolitische Pfeilspitzen beinhalteten, die ihre Ideologen geschickt mit pädagogischen Argumenten verschleierten“. Der Auslöser für diese Taktik lag natürlich in der Tatsache, dass die Mitglieder und Sympathisanten der Kommunistischen Partei zu dieser Zeit in der Illegalität agieren mussten, weswegen die Gründung der Lehrerbewegung eng mit Bemühungen verbunden waren, die Bewegung auf legaler Ebene zu organisieren.

Diese Bemühungen begannen am 29. 6. 1936, bei einer Massenversammlung in Maribor von linksorientierten angestellten und arbeitslosen Lehrern, und endeten am 5. 1. 1937, als eine Mariborer Arbeitsgruppe der Lehrerbewegung entstand und $\mathrm{zu}$ einer autonomen Organisationseinheit im Rahmen der Vereinigung der jugoslawischen Lehrerschaft wurde. Die Aktivitäten und Aktionen wurden hauptsächlich von den Mitgliedern des illegalen, linksorientierten Ausschusses der jüngsten Lehrer durchgeführt. Sie begannen fortschrittliche Bemühungen in und außerhalb Sloweniens zu studieren und neue Kontakte mit älteren, progressiven Lehrern zu suchen. In der pädagogischen Presse informierten sie über ihre Aktionen und propagierten ihre Sichtweisen mit dem Ziel, alle Lehrer in einer fortschrittlichen pädagogischen, sozialen und politischen Bewegung $\mathrm{zu}$ vereinen. Die Grundannahme, dass, um die Aufgaben der schulischen und außerschulischen Arbeit der Lehrer festzulegen, zuerst die konkreten sozioökonomischen Bedingungen des Lebens der untersten Klassen zu studieren sind, wollten sie mit einer Art öffentlichen Debatte (Umfrage) überprüfen, an der auch fortgeschrittene Studenten, Lehrer, Schriftsteller und Intellektuelle im Allgemeinen teilnahmen. Bei der Formulierung der programmbezogenen Forderungen hatte gerade Žgeč den größten Einfluss (Šegula, 1974).

Die Tatsache, dass in der Führung der Lehrerbewegung „die Lehrer Marxisten, Mitglieder und Unterstützer der Kommunistischen Partei“ (Remar, 1959, S. 186) waren bedeutet nicht, dass die Lehrerbewegung eine ideologisch und fachtheoretisch einheitliche Bewegung war oder politische Interessen im Vordergrund standen. Zweifellos beeinflusste ihre politische Ausrichtung stark die Bildung des pädagogischen Konzeptes, das eine Opposition gegenüber der universitären Kulturpädagogik in Ljubljana als auch - zumindest teilweise - gegenüber der reformpädagogisch orientierten Pädagogische Zentrale darstellte. Die aktive Beteiligung 
der Lehrerschaft an der empirischen Erforschung der psychophysischen Eigenschaften des slowenischen Kindes und der sozialen Bedingungen unter denen es lebt, trug an sich zu ihrer größeren sozialkritischen Sensibilität und erst in zweiter Linie zu ihrer politischen Einstellung bei. Strmčnik (1980, S. 84) argumentierte als Universitätsprofessor der Pädagogik in den 80er Jahren als der Marxismus die offizielle Gesellschaftsdoktrin war, die Aktivitäten der Ideologen des Lehrerbewegung folgendermaßen: „Durch die Einführung der Lehrerschaft in die marxistische Analyse der sozialen Realität haben sie erfolgreicher als mit anderen politischen Methoden die dialektisch-materialistische Auffassung der Gesellschaft und ihrer Haltung gegenüber der Bildung entwickelt, d. h., dass die Lebensbedingungen der Menschen einen großen Einfluss auf ihre Bildung haben. Sie wurden in die marxistische Literatur eingeführt und auf eine konkrete, aktive Weise für die ideelle und politische Position der Arbeiterklasse und der KPJ gewonnen.“

Es muss jedoch betont werden, dass die Lehrerbewegung der Kommunistischen Partei nichts unmittelbar schuldet oder dass sein pädagogisches Konzept nicht auf der Grundlage von Parteianweisungen entwickelt wurde. Im Gegenteil! $\mathrm{Zu}$ dieser Zeit war die Parteiführung gegenüber der Lehrerbewegung völlig gleichgültig, obwohl die Führung der Lehrerbewegung bestrebt war, ihre Arbeit in Einklang mit den Parteirichtlinien zu bringen. Jože Jurančič (damals als Aktivist der Lehrerbewegung) erinnerte sich Ende der 80er Jahre folgendermaßen daran: „Es war eine seltsame Beziehung, sie / die Parteiführung / nahmen unsere (Lehr-) Arbeit nicht ernst. Als ich bei ihnen in Ljubljana war, bei Tomšič und den anderen (bei der Parteiführung) sagten sie mir öfters ,Erzähl keine Märchen!', das wird später ganz anders sein“ (Autor, 1988, S. 45). Oskar Autor, der 1988 Jurančič interviewte, kommentierte das obige Zitat ironisch mit folgenden Worten: „Und es war wirklich ganz anders“ (Ebda). Es ging natürlich um die Sichtweise, die den konzeptuellen Unterschied zwischen der theoretisch durchdachten Tätigkeit linksorientierter Lehrer vor dem Krieg und der marxistischen pädagogischen Doktrin nach dem Krieg, als sich der theoretische Diskurs dem politischen Willen der regierenden Partei unterordnen musste, hervorheben möchte.

Vielleicht führte genau diese Haltung der Parteiführung (vor dem Krieg) dazu, dass das pädagogische Konzept der Lehrerbewegung eine theoretische Autonomie behielt und aus diesem Grunde für weltanschaulich und politisch unterschiedlich denkende Gelehrte bedingt (zumindest in einigen Elementen) annehmbar war. Eine konzentrierte Darstellung des pädagogischen Konzepts kann man in Martin Mencejs ${ }^{4}$ Buch Die Slowenische Schule und der Lehrer vor modernen Aufgaben (1937) nachlesen. Sein Text bringt in Bezug auf Žgeč's Bemühungen nicht viel Neues. Lediglich die Ideen der Reformpädagogik werden etwas in den Hintergrund gerückt, während die sozialen Aspekte der Erziehung etwas mehr in den Vordergrund treten. Während Žgeč das Erziehungsziel aus der Psychologie (Natur des Kindes) und Soziologie (Bedürfnisse der breitesten Schichten) entwickelte, ist für Mencej das einzige Kriterium zur Bestimmung des Erziehungsziels die aktuelle gesellschaftliche Situation bzw. die Ideen fortschrittlicher gesellschaftlicher Kräfte, die ein Abbild der „Forderungen und

\footnotetext{
${ }^{4}$ Er war der Initiator und führende Aktivist der Lehrerbewegung.
} 
Bedürfnisse breiter Arbeiterschichten, ihrer wirtschaftlichen, sozialpolitischen und kulturell-entwicklungsbezogenen Ausrichtung sind“" (Ebda, S. 23).

Neben einer stark sozialkritischen Ausrichtung wies die Lehrerbewegung ein weiteres Merkmal auf, das in diesem Kreis sehr stark betont wurde. Es ging um den Widerstand der Lehrer gegenüber der pädagogischen Theorie und deren Nachfrage nach praktisch einsetzbarer pädagogischer Literatur. Mencej stellte auf der Grundlage einer Umfrage, die mit 62 Lehrern durchgeführt wurde fest, dass ,alle, vom ersten bis zum letzten, den Bedarf an moderner Literatur für die praktische Arbeit in der Schule unterstreichen. Damit sind aber nicht nur zusätzliche Lehrmittel und Handbücher für die einzelnen Fächer in der Schule gemeint, sondern auch Anweisungen zur Beobachtung, für das Studium des Kindes und der Verhältnisse in denen es lebt!“ (Ebda, S. 47). Es stellt sich die Frage inwieweit die Behauptung stimmt, dass die Lehrer nicht nur an zusätzliche Lehrmittel und Handbücher dachten. Beobachtung, Studium des Kindes und der Verhältnisse, in denen es lebt, waren nämlich eine der grundlegenden Aufgaben der Lehrerbewegung, weswegen es durchaus möglich ist, dass dies die Folge der Annahme des Grundsatzprogramms ist und nicht ihrer Erkenntnis, dass sie genau solche Handbücher für ihre Arbeit brauchen. Ungeachtet dieses Zweifels haben aber relativ viele Lehrer beim Sammeln empirischer Daten über die sozialen Verhältnisse des slowenischen Kindes und seine körperliche Entwicklung mitgemacht. Damit wurde, wie Ende der 80er Jahre Mencej anführte, das Ziel der Ideologen der Lehrerbewegung, die Lehrer sollten durch das Kennenlernen der sozialen Unterschiede die Notwendigkeit des Klassenkampfs erkennen, verwirklicht (Autor, 1988, S. 43).

Der Forderung der Lehrerschaft nach möglichst direkt einsetzbarer Literatur versuchte Ledinek (ein weiterer wichtiger Aktivist der Lehrerbewegung) mit seinem Buch Meine Klasse (1939) nachzukommen. Er zeigte den Lehrkräften sehr detailliert, auf welche Art und Weise er die psychophysischen und sozialen Charakteristiken der Kinder in seiner Klasse untersucht hatte. Die Grundlage seiner empirischen Untersuchung war ein Kapitel aus den Beschreibungsbögen von Žgeč (Kindliche Meilensteine und seine Entwicklung in der Schulzeit) aus dem Jahr 1929, die vom 3. Selbstbildungskurs in Ljubljana bestätigt wurden. Der gesamte erste Teil des Buches ähnelt stark einem Forschungsbericht, der die statistischen Daten, die auf der Grundlage von Messungen physiologischer Charakteristiken, Intelligenzund Lerntests, Befragungen und Beobachtungen, Sammeln von Daten zu ihren Familien, der sozialen Situation und der Schuldokumentation (Lernerfolg) der Schüler gesammelt wurden, sehr genau darstellt. Obwohl er betonte, dass kein Lehrer ohne Kenntnisse der pädagogischen Theorie auskommt, nannte er als eine der Aufgaben des Lehrers als Praktiker, dass er die Theorie ständig überprüft und ergänzt bzw. dass er , /... / induktiv und deduktiv in die Theorie eindringt" (Ebda, S. 10). Letzteres auf der Grundlage der eigenen Analyse der Klasse als Gesamtheit, der einzelnen Schüler und der sie beeinflussenden Umgebung. Im Rahmen der pädagogischen und sozialen Ideen der Lehrerbewegung schuf ein solches Verständnis der Beziehung zwischen Theorie und Praxis eine neue Dimension des Verständnisses für die 
Funktion des Lehrers. Die Kenntnis der mentalen und physischen Eigenschaften des Kindes und der Klasse als Ganzes sollten es dem Lehrer ermöglichen, seine Arbeit den Fähigkeiten, Bedürfnissen und Wünschen der Kinder anzupassen, was die eigentlich grundlegende Forderung der Reformpädagogik ist. Der Lehrer ist jedoch wegen des Einflusses der sozialen Bedingungen, die das psychophysische Bild des Kindes maßgeblich stärker prägen als die Lehrer, machtlos. Dies bedeutet konkret, dass der pädagogische Optimismus, dem sich der Lehrer in der Reformpädagogik sowie in anderen pädagogischen Richtungen verpflichtet hat, hier in der sozialkritischen Pädagogik verloren geht (vgl. Protner, 2000).

Ledinek befürwortet in seinem Buch das didaktische Prinzip, dass erfolgreiches lernen und Bildung nur durch reichhaltige Erfahrungen der Lerner möglich ist. Damit übernahm er sowohl die Forderung der Reformpädagogik, wie auch der geisteswissenschaftlichen bzw. kulturellen Pädagogik. Seiner Meinung nach ist es nicht schwierig, bei einem normal intelligenten und in normalen Verhältnissen lebenden Schüler Erfahrungen und den Wunsch nach neuen Erfahrungen zu erreichen. Dies ist jedoch bei einem ,apathischen“ Kind schwerer $\mathrm{zu}$ erreichen und es bedarf ,greifbarerer Reize“. Aber selbst ein didaktisch und pädagogisch vollkommen an das Kind angepasster Unterricht muss sich letztendlich der Gesetzmäßigkeit, dass das soziale Umfeld entscheidend die kindliche Entwicklung beeinflusst, unterordnen: „Aber einem weniger talentierten Kind, das in schwierigen sozialen Verhältnissen lebt, oder von irgendwelchen negativen Störungen beeinflusst wird, kann auch das nicht helfen. Hier ist der Pädagoge machtlos“" (Ebda, S. 61).

Die für die Reformpädagogik charakteristische Idealisierung des Kindes und die für die Geistes- oder Kulturpädagogik charakteristische Idealisierung des Lehrers wird im sozialkritischen pädagogischen Konzepts der Lehrerbewegung durch eine wesentlich realistischere Sichtweise ersetzt. Das Kind wird zum Objekt des empirischen Studiums, das wesentlich von den sozialen Umständen, in denen es lebt, determiniert wird. In diesem Sinne sagt Ledinek: „Der Lehrer hat eine große Verantwortung in der Erziehung der Kinder, hat aber nicht die Kraft, in fünf Stunden pro Tag das auszugleichen, was ihnen zuhause nicht gegeben wurde und das zu korrigieren, was die Straße und Umgebung zerstört haben [...]“ (Ebda, S. 69).

\section{Fazit}

Die Tatsache, dass sich innerhalb des sozialkritischen pädagogischen Konzepts in Slowenien das Paradigma der empirischen Forschungsmethodik etabliert hatte, wirft eine Reihe von Fragen auf. Bereits in der Einleitung wurde die Dilemma hinsichtlich der Klassifizierung und Benennung des pädagogischen Konzepts, das hier beschrieben wurde, hervorgehoben. Auf der einen Seite entspricht das Konzept nicht in allen Details den Charakteristiken, die üblicherweise der materialistischen bzw. marxistischen pädagogischen Doktrin ${ }^{5}$

\footnotetext{
5 Die slowenischen Vertreter der sozialkritischen Pädagogik können am leichtesten der szientifischen pädagogischen Traditionslinie des materialistischen und sozialistischen Denkens, dem Kreis der Austromarxisten unter der Leitung von O. Glöckel und O. F. Kanitz zugeordnet werden (Vgl. Krüger, 1997, S. 102).
} 
zugeschrieben werden. Vor allem konnten keine Hinweise darauf gefunden werden, ob die Doktrin in der Zeit vor dem zweiten Weltkrieg empirische Forschungsmethoden einbezogen wurden. Auf der anderen Seite deckt das was im deutschsprachigen Raum weithin als „Empirische Erziehungswissenschaft“ angesehen wird, keineswegs die sozialempirischen Herangehensweisen $a b$, die von den linksorientierten Pädagogen in Slowenien praktiziert wurden. In Slowenien lassen sich die typischen Entwicklungsstadien der Empirischen Erziehungswissenschaft, die beispielweise Wulf (1983, S. 66ff.) von der anfänglichen experimentellen Pädagogik von W. A. Lay und E. Meumann, über die pädagogische Tatsachenforschung von Elsa und Peter Petersen bis zur deskriptiven Erziehungswissenschaft von A. Fischer und R. Lochner zeitlich ordnete, nicht nachweisen, obwohl die slowenische Pädagogik auch zu dieser Zeit noch immer unter starkem Einfluss pädagogischer Strömungen des deutschsprachigen Raums stand. Erst in der Nachkriegszeit könnte ein Kontakt der slowenischen Pädagogik mit den folgenden Entwicklungsstufen der Empirischen Erziehungswissenschaft bestätigt werden. Auch in Slowenien passierte in den 60er Jahren die realistische Wendung, aber ohne Bezug $\mathrm{zu}$ den Bemühungen Heinrich Roths, jedoch im Rahmen der Bemühungen der pädagogischen Wissenschaft zur empirischen Verifizierung (und Kritik) der Reformanstrengungen der Schulpolitik (Vgl. Medveš, 2015). Der kritische Rationalismus als fünfte Phase der Entwicklung der Empirischen Erziehungswissenschaft erfuhr in Slowenien keine nennenswerte Rezeption, ebenso wenig die Phasen der emanzipatorischen Bildungsforschung und der Handlungsforschung, obwohl der empirische Forschungsansatz generell stärker wurde und dieser Trend bis heute anhält.

Das erkenntnistheoretische Konzept der Sozialkritischen Pädagogik in Slowenien hängt wesentlich stärker von der Methodologie der soziologischen Forschung ab, als von der pädagogischen Methodologie. Leider konnte nicht festgestellt werden, welchen Modellen der empirischen soziologischen Erforschung des Kindesmilieus die slowenischen Pädagogen damals folgten. Dass es bei der Bearbeitung der empirischen Daten zu gravierenden methodischen Problemen kam, beweist der Kurs zur Statistik, der von der Schule für öffentliche Gesundheit in Zagreb in den Jahren 1939 und 1940 für Lehrer aus allen Teilen des damaligen Jugoslawien organisiert wurde (Babić-Weiner, 1974).

Wie eng die Idee der empirischen pädagogischen Forschung in der sozialkritischen Pädagogik, wie wir sie verstehen und vorgestellt haben, mit der linken politischen Option verknüpft war zeigt die Tatsache, dass die Organisatoren des Kurses systematisch linksorientierte Lehrer auswählten, die bereits Erfahrung mit empirischer Datensammlung hatten. Eine besondere Herausforderung stellt aber die These dar, dass auch die slowenische empirische Soziologie (und nicht nur die Pädagogik) innerhalb der vorgestellten Lehrerbewegung konstituiert wurde. 


\section{Literatur}

AUTOR, Oskar, 1988. Intervju z Jožetom Jurančičem in Martinom Mencejem. Maribor: Pedagoška fakulteta Maribor.

BABIĆ-WEINER, Vlatka, 1979. Drugi tečaj vitalne statistike. In: CICMIL, Obrad et al., Hrsg. Učesnici i svedoci. Zbornik sećanja o delatnosti naprednog učiteljstva Jugoslavije do 1941. godine. 2. Band. Društvo za izučavanje delatnosti naprednog učiteljstva Jugoslavije: Beograd, S. 287-289.

BERGANT, Milica, 1962. Uvod. In: BERGANT, Milica et al., Hrsg. Okolje in razvoj slovenskega otroka. Ljubljana: DZS, S. 5-9.

DOBERŠEK, Karel, 1929. Vpliv socialnih razmer na otroka v Prevaljah. Ljubljana: Slovenska šolska matica.

JURANČIČ, Jože, 1930. Iz šole za narod. Ljubljana: Slovenska šolska matica.

KANITZ, Otto Felix, 1925. Das proletarische Kind in der bürgerlichen Gesellschaft. Jena: Urania.

KRÜGER, Heinz-Hermann, 1997. Einführung in Theorien und Methoden der Erziehungswissenschaft. Opladen: Leske + Budrich. ISBN 3-8100-1542-3.

LEDINEK, Miloš, 1939. Moj razred. Maribor: Pedagoški tisk.

MENCEJ, Martin, 1937. Slovenska šola in učitelj pred sodobnimi nalogami. Maribor: Pedagoški tisk.

MEDVEŠ, Zdenko, 2015. Socialistična pedagogika, ujeta $v$ mit o pravičnosti enotne šole in kulturno hegemonijo = Socialist Pedagogy: Caught Between the Myth of the Fairness of the Unified School and Cultural Hegemony. Sodobna pedagogika. Jahrg. $66=132$, Nr. 2 , S. 14-37, 14-41. ISSN 0038-0474.

PAHOR, Jože, 1927. Samoizobraževalni tečaj v ljubljani. Popotnik. Jahrg. 49, Nr. 2, S. 4445.

PROTNER, Edvard, 2000. Pedagogika in izobraževanje učiteljev. Gorica: Educa. ISBN 9616039-42-3.

REMAR, Jože, 1959. Progresivna prizadevanja učiteljstva v okviru stanovske organizacije pred drugo svetovno vojno. Nova obzorja. Jahrg. 12, Nr. 4, S. 183-191.

SCHMIED-KOWARZIK, Wolfdietrich, 1983. Materialistische Erziehungstheorie. In: LENZEN, Dieter und MOLLENHAUER, Klaus, Hrsg. Theorien und Grundbegriffe der Erziehungswissenschaft. Bd. 1 der Enzyklopädie Erziehungswissenschaft. Stuttgart: KlettCotta, S. 101-116. ISBN 3129399542.

STRMČNIK, France, 1980. Zveza društev pedagoških delavcev Slovenije v luči svoje pozitivne pedagoške tradicije. Sodobna pedagogika. Jhrg., 31, Nr. 1-2, 3-4, S. 15-35, 81-87. ŠEGULA, Pavle, 1974. Borba za stvaranje jedinstvenog progresivnog učiteljskog pokreta u Sloveniji. In: CICMIL, Obrad et al., Hrsg. Učesnici i svedoci; Zbornik sećanja o delatnosti naprednog učiteljstva Jugoslavije do 1941. godine. 1. Band. Beograd: Društvo za izučavanje delatnosti naprednog učiteljstva Jugoslavije, S. 351-369.

ŠULIGOJ, Avgust, 1928. Stanovanjske razmere in njihov vpliv na otroka. Popotnik. Jhrg. 50, Nr. 3-4, S. 89-91. 
ŠULIGOJ, Avgust, 1929. Vpliv gospodarskih in stanovanjskih razmer na razvoj otroka. Popotnik. Jhrg. 50, Nr. 7, S. 210-212.

TANCER, Mladen, Hrsg., 1991. Dr. Franjo Žgeč; socialno-pedagoški reformator na Slovenskem. Maribor: Univerza v Mariboru, Pedagoška fakulteta, Raziskovalni institut. TENORTH, Heinz-Elmar, 1992. Materialistisch orientierte Pädagogik. In: PETERSEN, Jörg und REINERT, Gerd-Bodo, Hrsg. Pädagogische Konzeptionen. Donauwörth: Auer, S. 201220. ISBN-10 3403022080.

WULF, Christoph, 1983. Theorien und Konzepte der Erziehungswissenschaft, 3. Aufl., München: Juventa.

ŽGEČ, Fran, 1923. Problemi vzgoje najširših plasti našega naroda. Ljubljana: Udruženje Jugoslovenskega učiteljstva - Poverjeništvo Ljubljana.

ŽGEČ, Fran, 1926. Razvoj otroka v šolski dobi. In: ŠILIH, Gustav, Hrsg. Pedagoški zbornik. Ljubljana: Slovenska šolska matica, S. 46-112.

ŽGEČ, Fran, 1928. „Idejni in praktični program Pedagoške centrale“ v Mariboru. In: ŠILIH, Goran, Hrsg. Pedagoški zbornik. Ljubljana: Slovenska šolska matica, S. 145-150. 